\title{
Phase-slip phenomena in NbN superconducting nanowires with leads
}

\author{
A. K. Elmurodov and F. M. Peeters* \\ Department of Physics, University of Antwerp, Groenenborgerlaan 171, B-2020 Antwerpen, Belgium
}

D. Y. Vodolazov

Department of Physics, Nizhny Novgorod University, 603600 Nizhny Novgorod, Russia

S. Michotte, S. Adam, F. de Menten de Horne, and L. Piraux

Universite Catholique de Louvain (UCL), Place Croix du Sud 1, B-1348 Louvain-la-Neuve, Belgium

$$
\text { D. Lucot and D. Mailly }
$$

Laboratoire de photonique et de nanostructures (LPN-CNRS), route de Nozay, F-91460 Marcoussis, France

(Received 15 July 2008; revised manuscript received 28 October 2008; published 31 December 2008)

\begin{abstract}
Transport properties of a superconducting $\mathrm{NbN}$ nanowire are studied experimentally and theoretically. Different attached leads (superconducting contacts) allowed us to measure current-voltage $(I-V)$ characteristics of different segments of the wire independently. The experimental results show that with increasing the length of the segment the number of jumps in the $I$ - $V$ curve increases indicating an increasing number of phase-slip phenomena. The system shows a clear hysteresis in the direction of the current sweep, the size of which depends on the length of the superconducting segment. The interpretation of the experimental results is supported by theoretical simulations that are based on the time-dependent Ginzburg-Landau theory, the heat equation has been included in the Ginzbur-Landau theory.
\end{abstract}

DOI: 10.1103/PhysRevB.78.214519

PACS number(s): 74.25.Sv, 74.25.Fy

\section{INTRODUCTION}

It is known that phase-slip centers (PSC) occur in the resistive state of long quasi-one-dimensional channels. ${ }^{1}$ The succession of critical currents and intermediate states has been investigated on "one-dimensional" samples, e.g., whiskers $^{2}$ and microbridges ${ }^{3}$ exhibiting phase-slip centers. ${ }^{4}$ A dissipative state has a resistance significantly below the normal-state value. In one or more areas along the wire a phase slip is created which is a region (with size about coherence length $\xi$ ) of dynamically suppressed order parameter $\xi$ and surrounded by diffusive tails where the quasiparticles and Cooper pairs have different electrochemical potentials (providing a local charge imbalance). The diffusion length associated with the relaxation time for this specific nonequilibrium effect determines the effective resistance of the phase-slip center.

Recently experiments on superconducting nanowires ${ }^{5}$ have attracted renewed attention on the electrical and magnetic manifestations of thermal and quantum fluctuations. The successive nucleation of PSC leads to the appearance of a stairlike structure in the $I-V$ characteristics. ${ }^{6,7}$ The existence of phase-slip centers was confirmed in low-temperature superconductors. ${ }^{8}$ Recently phase-slip centers were observed $^{9}$ in the presence of an external applied magnetic field. It was shown in Ref. 10 that with attaching many lowresistance Ohmic contacts along the electrodeposited Sn nanowires one can perform electrical measurements on different segments of the superconducting wire. It is also often pointed out that phase-slip lines in wide films are similar to phase-slip center in narrow films. ${ }^{11,12}$

In this paper we present experimental results on currentvoltage characteristics of a mesoscopic superconducting $\mathrm{NbN}$ wire with many superconducting contacts (see Fig. 1).
In this geometry, the contacts play a very important role because they make it possible to study experimentally the size effect on the phase-slip phenomena. The effect of the sample length on the formation of the phase-slip centers is studied within the time-dependent Ginzburg-Landau (GL) theory. The GL equation is coupled to the heat diffusion equation because of inevitable heat dissipation, which can lead to a local transition to the normal state.

\section{EXPERIMENTAL RESULTS}

The sample was fabricated as follows: thin $\mathrm{NbN}$ films were deposited from a $\mathrm{Nb}$ target by $\mathrm{DC}$ magnetron sputtering

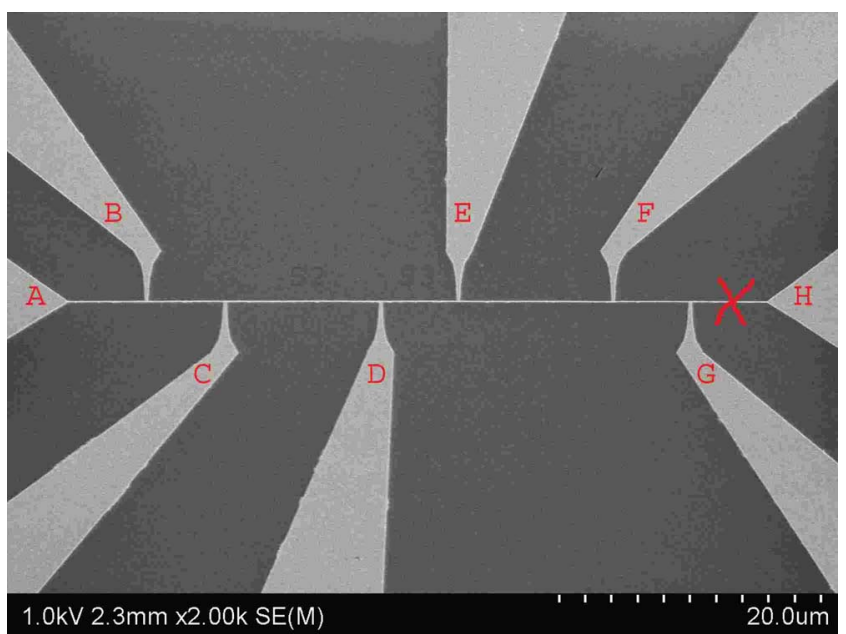

FIG. 1. (Color online) A scanning electron microscopy image of a $\mathrm{NbN}$ nanowire with attached current and voltage electrodes. Segment GH is broken. 

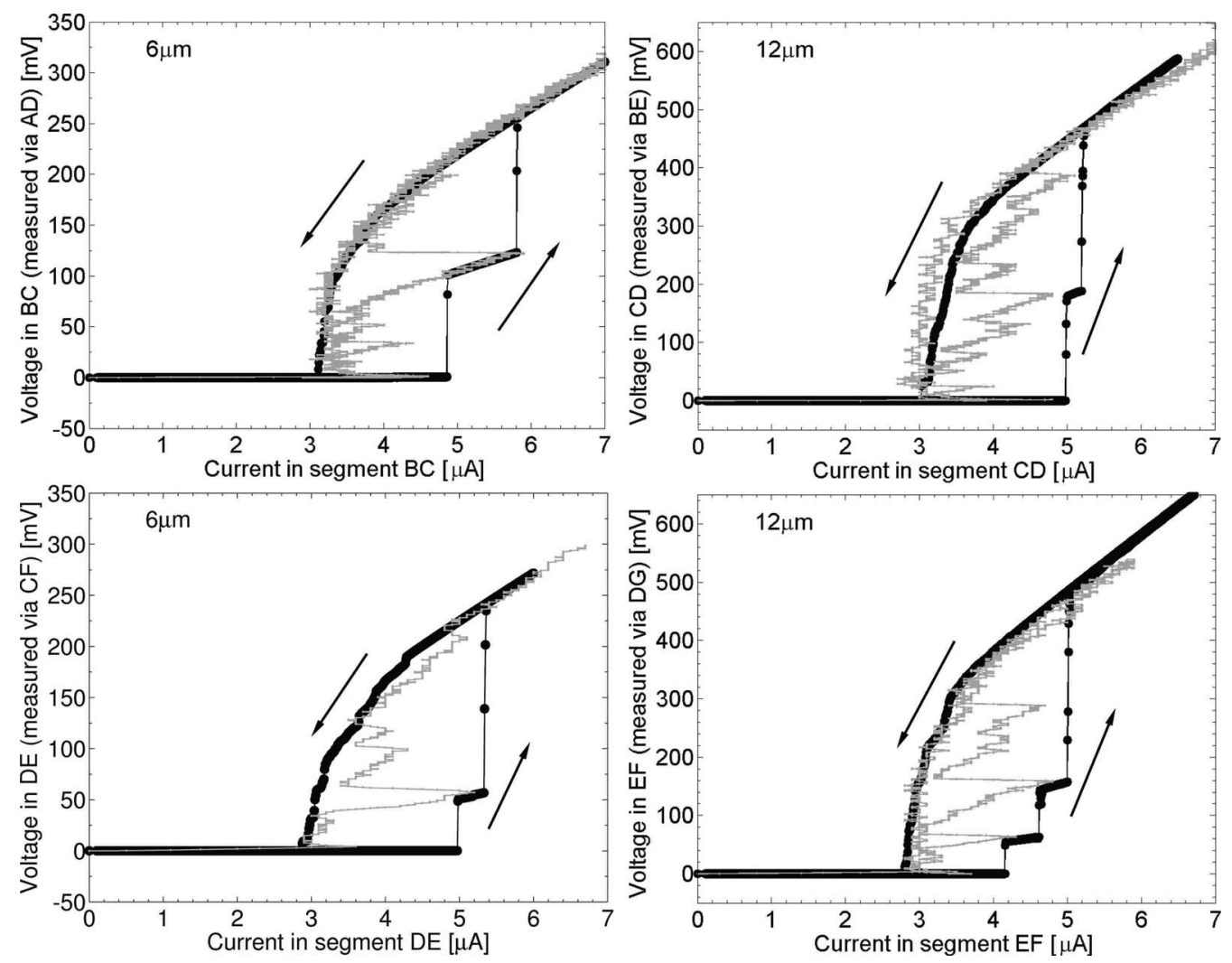

FIG. 2. Voltage-current characteristic of BC, CD, DE, and EF segments (6 $\mu \mathrm{m}$ or $12 \mu \mathrm{m}$ long, $70 \mathrm{~nm}$ wide, and $75 \mathrm{~nm}$ thick; four contacts) of the sample (see Fig. 1) under current drive (black curve) or voltage drive (gray curve) condition. Base temperature is $4.2 \mathrm{~K}$ and no external magnetic field is applied.

on a $\mathrm{Si} / \mathrm{SiO}_{2}$ substrate at room temperature in a $8.55 \mathrm{mTorr}$ $\mathrm{Ar} / \mathrm{N}_{2}$ atmosphere [gas flow: 38.2 and 2.43 SCCM (SCCM denotes cubic centimeter per minute at STP), respectively]. This process yields typically $T_{c}=10 \mathrm{~K}$ and $\xi \sim 5 \mathrm{~nm}$ for film thickness of a few tens of $\mathrm{nm}$. The $\mathrm{NbN}$ films were patterned to form the structure shown in Fig. 1 by SF6 reactive ion etching using a $10-20 \mathrm{~nm}$ thick $\mathrm{Al}_{2} \mathrm{O}_{3}$ mask. The pressure and the bias for the $\mathrm{SF} 6$ etch are chosen such that the $\mathrm{Al}_{2} \mathrm{O}_{3}$ is not damaged. This $\mathrm{Al}_{2} \mathrm{O}_{3}$ mask is subsequently removed with $\mathrm{NaOH}$. The multicontacts are thus of the same nature than the strip under study and are labeled A,B,C,... as shown in Fig. 1 from left to right. Samples were cooled to helium boiling temperature in a cryostat and each measurement line was filtered using pi filters. Current or voltage bias was applied to each segment using a four contact configuration.

Figures 2 and 3 present the current-voltage properties of each segment (respectively, 6 and 12 or $36 \mu \mathrm{m}$ long) of the wire. Their normal-state resistance just above $T_{c}$ is quite high $(8.5 \mathrm{k} \Omega / \mu \mathrm{m})$, which is characteristic for such a hard superconductor. As a consequence there is Joule heating but the relatively good cooling from the leads and the substrate allows to avoid the formation of a self-spreading hotspot ${ }^{3}$ in current bias when the critical current is reached. Rather with current sweep up, resistive domains appear successively and they can be stabilized in a wide current range. Comparing Figs. 2 and 3, we also notice that the total number of these resistive domains scales with the length of the segment. Applying the voltage (gray curves in Figs. 2 and 3), we see that even more of such nonequilibrium regions can be formed in the segment than the current drive suggest. This indicates the possibility of obtaining different number of such normal regions simultaneously. In a current sweep down a continuous behavior is found without any jump between different resistive states.

Figure 4 shows the $I-V$ curve of another, shorter, segment, measured with only two contacts. It has a smaller normalstate resistance $(5.3 \mathrm{k} \Omega / \mu \mathrm{m})$. The resistance of the measuring lines (wiring filtering) being small (30 $\Omega$ each), the initial increase in the voltage before the first critical current mainly results from a uniform flow of Abrikosov vortices moving transversally to the segment. Due to the Lorentz

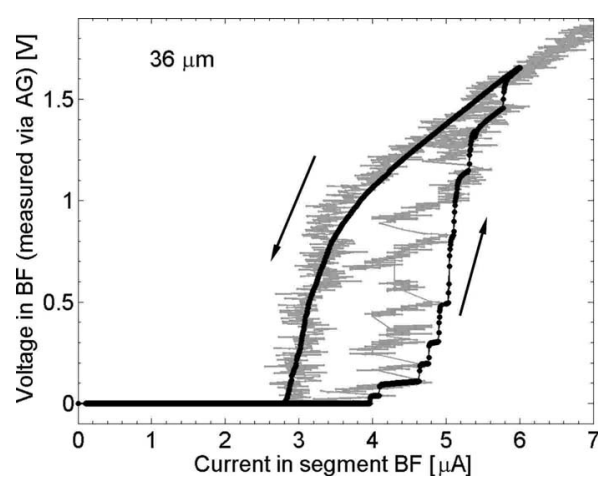

FIG. 3. The same as Fig. 2 but for the segment BF (36 $\mu \mathrm{m}$ long, $70 \mathrm{~nm}$ wide, and $75 \mathrm{~nm}$ thick) in Fig. 1. 


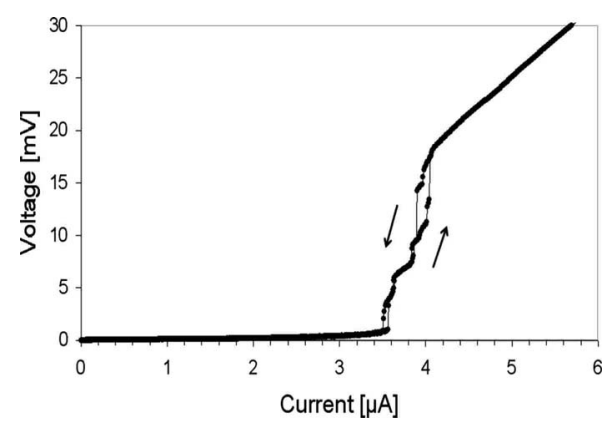

FIG. 4. Voltage-current characteristic of the $\mathrm{NbN}$ segment (1 $\mu \mathrm{m}$ long, $60 \mathrm{~nm}$ wide, and $50 \mathrm{~nm}$ thick) under current bias condition with two contacts. Base temperature is $4.2 \mathrm{~K}$ and there is no external magnetic field.

force that acts on the trapped or self-induced vortices, this flow of Abrikosov vortices (so-called flux flow regime) is preceding the formation of the resistive domains, as this is sometimes observed. ${ }^{13}$ The high number of successive resistive domains in this smaller and shorter segment is probably linked to a better cooling (merely due to good thermal connection with leads) combined with a smaller Joule effect.

Due to the regularity of our samples, as illustrated in Fig. 1 , we feel that heating cannot be responsible for the formation of these various domains (although it probably affects them). Indeed, pure hotspots would tend to coalesce as the current is raised. Therefore, the formation of phase-slip lines is a natural mechanism to think of in view of our results, even if they are observed here far from the critical temperature. Given the similarities between phase-slip line and phase-slip center, this is not surprising since phase-slip centers have also been observed far from $T_{c}$. Therefore, it makes sense to compare these results to the theoretical model of phase-slip center in which the heating effect is included.

\section{THEORETICAL MODEL}

For our theoretical study we use the generalized timedependent Ginzburg-Landau (TDGL) (Refs. 14 and 15) equation to describe the order parameter in the wire:

$$
\begin{aligned}
& \frac{u}{\sqrt{1+\gamma^{2}|\psi|^{2}}}\left(\frac{\partial}{\partial t}+i \varphi+\frac{\gamma^{2}}{2} \frac{\partial|\psi|^{2}}{\partial t}\right) \psi=(\nabla-i \mathbf{A})^{2} \psi+(1-T \\
& \left.-|\psi|^{2}\right) \psi
\end{aligned}
$$

which is supplemented with the equation for the electrostatic potential

$$
\Delta \varphi=\operatorname{div}\left\{\operatorname{Im}\left[\psi^{*}(\nabla-i \mathbf{A}) \psi\right]\right\} .
$$

Here there is an explicit inclusion of the time relaxation $\tau_{\epsilon}$ (through the coefficient $\gamma=2 \tau_{\epsilon} \Delta_{0} / \hbar$ ) for the nonequilibrium quasiparticle distribution due to interaction and $\Delta_{0}$ $=4 k_{B} T_{c} / u^{1 / 2} \pi$ is the "effective" value of the order parameter at $T=0$. In Eq. (1) all the physical quantities (order parameter $\psi=|\psi| e^{i \phi}$, electrostatic potential $\left.\varphi\right)$ are measured in dimensionless units: the momentum of superconducting condensate $p=\nabla \phi$ is scaled by the unit $\Phi_{0} /[2 \pi \xi(0)]$ (where $\Phi_{0}$ is the quantum of magnetic flux), the order parameter is in units of

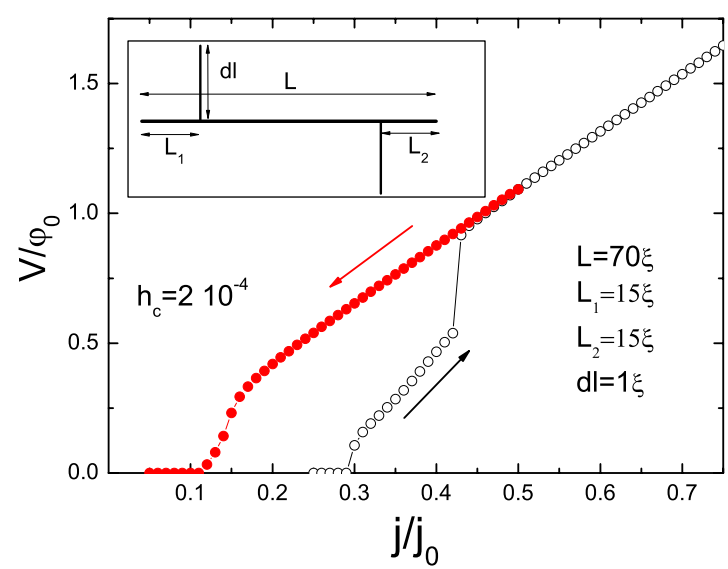

FIG. 5. (Color online) Current-voltage characteristic of a superconducting wire of length $L=70 \xi$ with two attached leads of size $d l=1 \xi$ for increased (open circles) and decreased (filled circles) applied currents. The distances from the two edges of the wire to the leads are $L_{1}=L_{2}=15 \xi$. The inset shows a schematic view of our model system.

$\Delta_{0}$, and the coordinates are in units of the coherence length $\xi(0)=\left(8 k_{B} T_{c} / \pi \hbar D\right)^{-1 / 2}(D$ is the diffusion constant). In these units the current density is scaled with $j_{0}$ $=\sigma_{n} \hbar / 2 e \tau_{\mathrm{GL}}(0) \xi(0)$, the time is in units of the GinzburgLandau relaxation time $\tau_{\mathrm{GL}}(0)=\pi \hbar / 8 k_{B} T_{c} u$, and the electrostatic potential $\varphi$ is in units of $\varphi_{0}=\hbar / 2 e \tau_{\mathrm{GL}}(0)\left(\sigma_{n}\right.$ is the normal-state conductivity). The vector potential is equal to zero because there is no applied magnetic field, and selfinduced effects are small for our system.

To simulate the real experimental situation we considered a superconducting wire with two attached leads, as illustrated in the inset of Fig. 5. Here we present our results obtained for the temperature $T=0.9 T_{c}$ and for the parameters $u=5.79$ and $\gamma=40 .{ }^{14}$ To simulate a real experimental situation we use "bridge" boundary conditions $|\psi(-L / 2)|=|\psi(L / 2)|=1$, $\psi( \pm L / 2, t+d t)=\psi( \pm L / 2, t) \exp ^{-i \varphi[\psi( \pm L / 2) d t]}$, and implemented the boundary condition $\partial \psi / \partial x=0$ at the contacts and $\varphi(-L / 2, t)=0$. Initial conditions were $|\psi|=1$ and $\varphi=0$. The behavior of the system is studied on a large time scale when time-averaged values no longer depend on time.

The superconducting wire is thermally conducting: since the electronic contribution to this thermal conductivity is very small for $\mathrm{NbN}$ at low $T$, the thermal conductivity is close to its value in the normal state. Thus the wire as well as the substrate will carry away the generated heat. Therefore, we have to couple the TDGL equation to the temperature diffusion equation. We solved the equation for the temperature distribution in the sample:

$$
C_{\mathrm{eff}} \frac{\partial T}{\partial t}=k_{\mathrm{eff}} \Delta T+j_{n}^{2}-h_{c}\left(T-T_{0}\right),
$$

where $\quad C_{\mathrm{eff}}=\left(D_{s} C_{s} / d_{f}+C_{f}\right) T_{c} \sigma_{n} / \tau_{\mathrm{GL}}(0) j_{0}^{2}, \quad k_{\mathrm{eff}}=\left(D_{s} k_{s} / d_{f}\right.$ $\left.+k_{f}\right) T_{c} \sigma_{n} / \xi^{2} j_{0}^{2}, h_{c}=k_{s} T_{c} \sigma_{n} / D_{c} d_{f} j_{0}^{2}$, the heat transfer coefficient $h_{c}$ governs the heat removal from the sample, and $C_{s}$, $C_{f}, k_{s}$, and $k_{f}$ are heat capacity and heat conductivity of the substrate (subscript $s$ ) and film and sample (subscript $f$ ), respectively. Here we used a model for the temperature distri- 

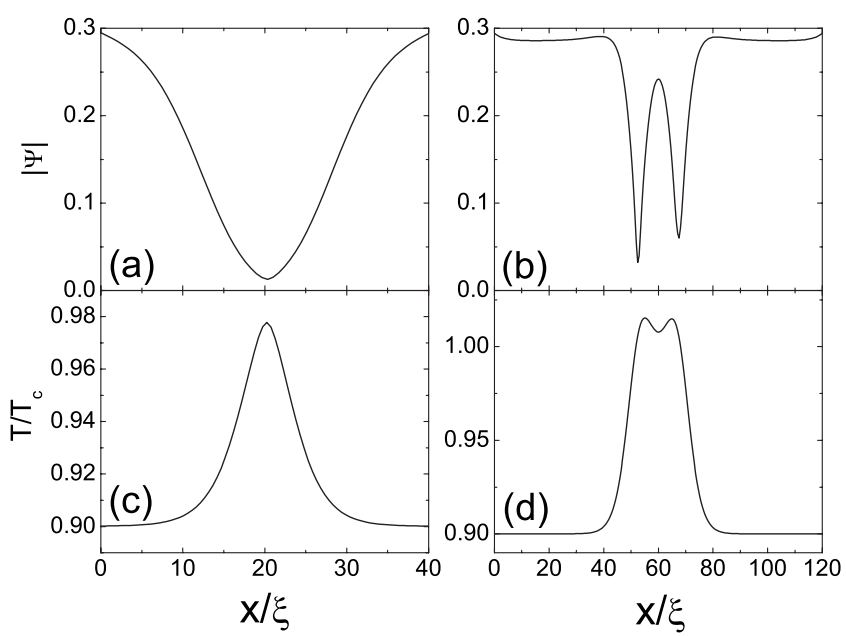

FIG. 6. The time average modulus of the [(a) and (b)] order parameter $|\psi|$ and $[(\mathrm{c})$ and (d)] temperature between the two contacts $L_{1}=L_{2}=15 \xi$. The length of the wire is [(a) and (c) $L=70 \xi$ and [(b) and (d)] $L=150 \xi$; the applied current density is [(a) and (c)] $j=0.3 j_{0}$ and $[(\mathrm{b})$ and $(\mathrm{d})] j=0.4 j_{0}$.

bution in thin superconducting films as was previously discussed in details in Ref. 16, and we assumed that the thickness of the substrate and film, $D_{c}+d_{f}$, is much smaller than the healing length $\Lambda_{h}=\sqrt{k_{\mathrm{eff}} / h} \gg D_{s}+d_{f}$. If $D_{s} C_{s} / d_{f}$ $\ll C_{f}$ and $D_{s} k_{s} / d_{f} \ll k_{f}$, we can use the Wiedermann-Franz law as an estimate for $C_{f}$ and $k_{f}$, and we obtain for $C_{\text {eff }}$ $=\pi^{3} / 48 \simeq 0.65$ and $k_{\text {eff }}=\pi^{4} / 48 u^{2} \simeq 0.06$ at temperature close to $T_{c}$. Because of the uncertainty in the actual values of $C_{s}$ and $k_{s}$ we used following values: $C_{\text {eff }}=2.0$ and $k_{\text {eff }}=0.0003$. We checked that our results only weakly depend on our choice of $C_{\text {eff }}$ and $k_{\text {eff- }}$. We also put $A=0$ in Eqs. (1) and (2) because we considered the one-dimensional model, in which the effect of the self-induced magnetic field is negligible and we assume that no external magnetic field is applied. ${ }^{17}$

\section{NUMERICAL RESULTS}

In this chapter we present our theoretical results about transport properties of the system in the current driven regime. Let us first consider the effect of the wire length on the $I-V$ characteristics of the system. Figure 5 shows the $I-V$ curve of the sample with length $L=70 \xi$ for both increasing (open symbols) and decreasing (filled symbols) currents. With increasing the applied current the system switches to the first resistive state with a jump in the voltage at the upper critical current $j_{c 2}=0.3 j_{0}$. At this value of the current one phase-slip enters the sample leading to finite resistance of the sample. Because of the chosen geometry the phase-slip center appears always in the center of the sample where the order parameter is minimal [see Fig. 6(a)]. Because of the heat transfer the local temperature increases in the phase-slip centers [see Fig. 6(c)]. With further increasing the current the frequency of the order parameter oscillations increases leading to a larger time average voltage over the wire. Finally one observes the jump to the normal state and destruction of superconductivity over the whole sample.

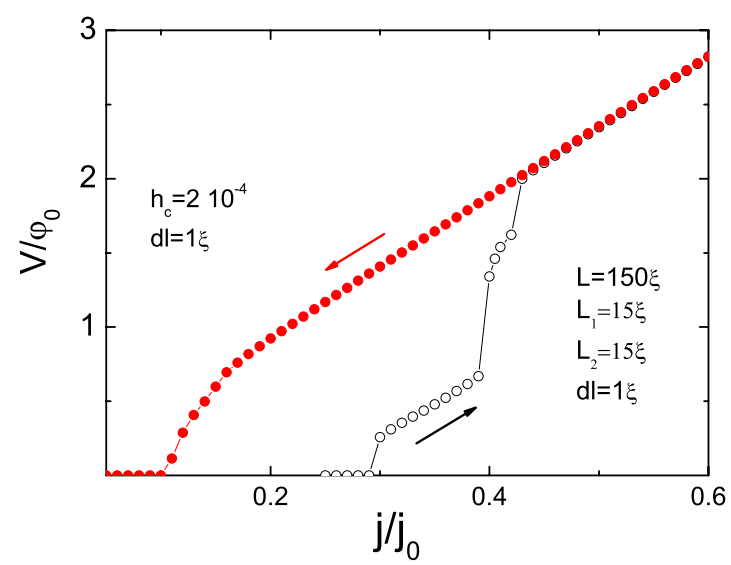

FIG. 7. (Color online) The same as Fig. 1 but now for a longer wire with length $L=150 \xi$.

If we start from the normal state and decrease the current the superconducting state is nucleated at the current $j_{c 1}$ $=0.11 j_{0}$, which is much smaller than $j_{c 2}$, i.e., the system exhibits a clear hysteresis similar to what we obtained experimentally (see Fig. 2). For decreasing current the superconducting state is not recovered for current $j_{c 1}<j<j_{c 2}$ because of the high resistance which leads to larger heating of the sample. Note that no jumps in the $I-V$ curve is found in the current sweep down regime, which agrees with the experimental results.

Figure 7 shows the $I-V$ curve of a longer wire with size $L=150 \xi$ in the two current sweep regimes. The other parameters of the sample are the same as for the one in Fig. 5. It is seen from this figure that the number of voltage jumps in the $I-V$ curve increases with increasing the length of the wire, which is in good agreement with our experiment (see Figs. 2 and 3). The latter is due to the fact that a longer wire can accommodate a larger number of phase-slip centers while remaining in the superconducting state. As shown in Figs. 6(b) and 6(d), phase-slip centers appear in two places in the sample. Although the number of phase-slip centers increased with increasing the length of the wire, the second critical current $j_{c 2}$ and the current corresponding to the superconducting-normal state transition remain the same. However, the first critical current $j_{c 1}$ slightly decreases with increasing the size of the wire (compare with experimental results in Figs. 2 and 3). With current sweep down there are again no voltage jumps in the $I-V$ curve (compare with Figs. 2 and 3). As was shown in Ref. 6 the voltage jump at $j$ $=j_{c 1}$ depends on temperature roughly as $\Delta V \sim \hbar /\left(e \tau_{\epsilon}\right)(1$ $\left.-T / T_{c}\right)^{1 / 2}$ near the critical temperature and hence local heating strongly decreases this voltage jump.

Next we study the influence of the heat transfer coefficient $h_{c}$ to the obtained results. As an example, we plotted in Fig. 8 the $I-V$ curve of the sample considered in Fig. 7 but for two different heat transfer coefficients that amount to $h_{c}=2 \times 10^{-3},\left(h_{c}=2 \times 10^{-2}\right)$ which is ten (100) times larger than the one in Fig. 7. It is seen that the behavior of the curve for increasing current is the same as in Fig. 7. However, in this case the curve for decreasing current changes drastically: jumps appear indicating the presence of phase-slip centers in the sample. If the heat transfer coefficient increases by 100 


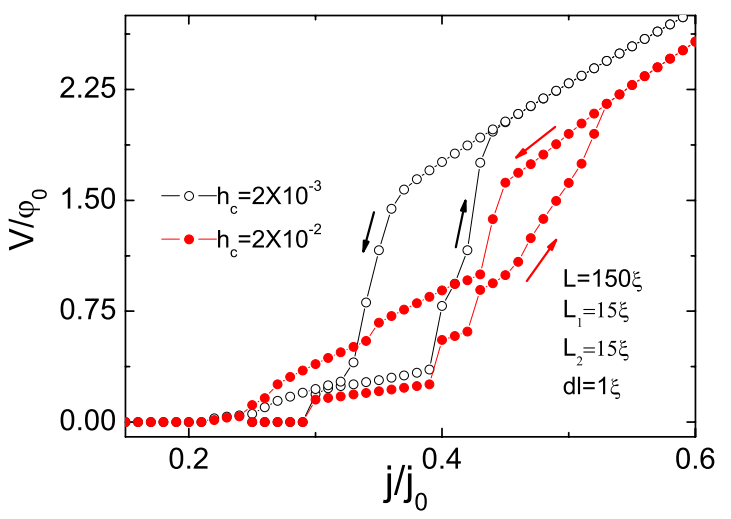

FIG. 8. (Color online) The same as Fig. 7 but now for heating transfer coefficients $h_{c}=2 \cdot 10^{-3}$ (open circles) and $h_{c}=2 \cdot 10^{-2}$ (filled circles).

times the one in Fig. 7, first, the number of voltage jumps in the current sweep up regime increases considerably. Second, the finite size steps in the $I-V$ characteristics are found even for decreasing currents. Finally, the first critical current $j_{c 1}$ considerably increases with increasing $h_{c}$. The reason for such changes is that when we increase $h_{c}$, the heat removal from the system increases and there is practically no local temperature increase due to the formation of phase-slip centers. As a result the decay length of the electric field is small and there is place in the wire for many phase-slip centers.

\section{CONCLUSION}

We have studied transport properties of a superconducting $\mathrm{NbN}$ wire with many contacts attached allowing us to per- form transport measurements in different segments of the wire. The system exhibits clear hysteresis with finite jumps in the $I-V$ curve. Each jump corresponds to phase-slip lines entering the sample, which was confirmed by numerical simulations using the time-dependent GL theory. The number and size of these jumps strongly depends both on the dimensions (i.e., length of the wire) and the heat transfer properties of the system. The value of the superconducting-normal transition current increases with increasing both the sample size and the heat transfer coefficient, whereas the critical current for the entrance of the first phase-slip remains the same. Our numerical calculations show that heating not always leads to complete destruction of superconductivity (as for example was supposed in Ref. 18), but instead may provide changes in superconducting properties due to local increase in temperature. For example the size of the phase slip center increases with temperature and it can qualitatively explain the presented experimental results.

\section{ACKNOWLEDGMENTS}

This work was supported by the Flemish Science Foundation (FWO-Vl), the Belgian Science Policy (Interuniversity Attraction Pole Program (P6/42)-Belgian State-Belgian Science Policy). D.Y.V. acknowledges support from the Dynasty Foundation. S.A. is a research fellow of the FNRS Belgium, F.M.H. acknowledges financial support from the FRIA, and S.M. is a postdoctoral researcher of the FNRS.

\footnotetext{
*francois.peeters@ua.ac.be

${ }^{1}$ R. Tidecks, Springer Tracts in Modern Physics (Springer, Berlin, 1990), Vol. 121.

${ }^{2}$ J. D. Meyer, Appl. Phys. (Berlin) 2, 303 (1973).

${ }^{3}$ W. J. Skocpol, M. R. Beasley, and M. Thinkham, Low Temp. Phys. 16, 145 (1974).

${ }^{4}$ G. J. Dolan and L. D. Jackel, Phys. Rev. Lett. 39, 1628 (1977).

${ }^{5}$ A. Bezryadin, C. N. Lau, and M. Thinkham, Nature (London) 404, 971 (2000).

${ }^{6}$ S. Michotte, S. Matefi-Tempfli, L. Piraux, D. Y. Vodolazov, and F. M. Peeters, Phys. Rev. B 69, 094512 (2004).

${ }^{7}$ D. Y. Vodolazov, F. M. Peeters, L. Piraux, S. Matefi-Tempfli, and S. Michotte, Phys. Rev. Lett. 91, 157001 (2003).

${ }^{8}$ Z. Zhou, R. Jin, G. Eres, D. Mandrus, V. Barzykin, P. Schlottmann, Y. S. Hor, Z. Xiao, and J. F. Mitchell, Phys. Rev. B 76, 104511 (2007).

${ }^{9}$ A. Falk, M. M. Deshmukh, A. L. Prieto, J. J. Urban, A. Jonas, and H. Park, Phys. Rev. B 75, 020501(R) (2007).
}

${ }^{10}$ D. Lucot, F. Pierre, D. Mailly, K. Yu-Zhang, and S. Michotte, F. de Menten de Horne, and L. Piraux, Appl. Phys. Lett. 91, 042502 (2007).

${ }^{11}$ V. G. Volotskaya, I. M. Dmitrenko, L. E. Musienko, and A. G. Sivakov, J. Low Temp. Phys. 10, 179 (1984).

${ }^{12}$ V. I. Kuznetsov and V. A. Tulin, JETP Lett. 61, 1026 (1995).

${ }^{13}$ V. M. Dmitriev, I. V. Zolochevskii, T. V. Salenkova, and E. V. Khristenko, Low Temp. Phys. 31, 127 (2005).

${ }^{14}$ L. Kramer and R. J. Watts-Tobin, Phys. Rev. Lett. 40, 1041 (1978).

${ }^{15}$ R. J. Watts-Tobin, Y. J. Krahenbuhl, and L. Kramer, Low Temp. Phys. 42, 459 (1981).

${ }^{16}$ A. V. Gurevich and R. G. Mints, Rev. Mod. Phys. 59, 941 (1987).

${ }^{17}$ D. Y. Vodolazov, A. Elmuradov, and F. M. Peeters, Phys. Rev. B 72, 134509 (2005).

${ }^{18}$ M. Tinkham, J. U. Free, C. N. Lau, and N. Markovic, Phys. Rev. B 68, 134515 (2003). 